\title{
Angle-restricted sets and zero-free regions for the permanent
}

Pavel Etingof (MIT)

\section{To the memory of E. V. Glivenko}

\section{INTRODUCTION}

A subset $S \subset \mathbb{C}^{*}:=\mathbb{C} \backslash\{0\}$ is called a zero-free region for the permanent if the permanent of a square matrix (of any size $n$ ) with entries in $S$ is necessarily nonzero. The motivation for studying such regions comes from the work of A. Barvinok ([B1]), where he shows that the logarithm of the permanent of such a matrix can be computed within error $\varepsilon$ in quasi-polynomial time $n^{O(\log n-\log \varepsilon)}$ (while the problem of efficient computation of general permanents is hopelessly hard). Namely, it is shown in [B1] that the disk $|z-1| \leq 1 / 2$ and a certain family of rectangles are zero-free regions, which enables efficient approximate computation of permanents of matrices with entries from these regions 1

The goal of this note is to give a systematic method of constructing zero-free regions for the permanent. We do so by refining the approach of [B1] using the clever observation that a certain restriction on a set $S$ involving angles implies zero-freeness ([B1]); we call sets satisfying this requirement angle-restricted. This allows us to reduce the question to a low-dimensional geometry problem (notably, independent of the size of the matrix!), which can then be solved more or less explicitly. We give a number of examples, improving some results of [B1]. This technique also applies to more general problems of a similar kind, discussed in [B2].

Acknowledgements. This paper was inspired by the Simons lectures of A. Barvinok at MIT in April 2019; namely, it is a (partial) solution of a "homework problem" given in one of these lectures. I am very grateful to A. Barvinok for useful discussions, suggestions and encouragement. I am also very indebted to two anonymous referees for thorough reading of the paper and very useful comments and corrections.

I dedicate this paper to the memory of my teacher Elena Valerievna Glivenko, Professor of Applied Mathematics at the Moscow Oil and Gas Institute, where I was a student in the late 1980s. Her teaching and care made an enormous difference for all of us.

\footnotetext{
${ }^{1}$ We note that a (randomized) efficient algorithm for computing the permanent of a matrix with nonnegative entries was proposed earlier in [JSV].
} 


\section{Definition AND BASIC PROperties of ANGLE-RESTRICTED SETS}

For $u, v \in \mathbb{C}^{*}$ let $\alpha(u, v) \in[0, \pi]$ be the angle between $u$ and $v$. Let $\theta, \phi \in(0,2 \pi / 3)$. Note that if $u_{1}, \ldots, u_{n} \in \mathbb{C}^{*}$ are such that $\alpha\left(u_{i}, u_{j}\right) \leq \theta$ then there exists $\lambda \in \mathbb{C}^{*}$ such that $\left|\arg \left(\lambda u_{i}\right)\right| \leq \theta / 2$ for all $i$ (where we agree that $\arg (z)$ takes values in $(-\pi, \pi])$.

Definition 2.1. (i) We denote by $A_{\theta, \phi}$ the set of subsets $S \subset \mathbb{C}^{*}$ such that for any $u_{1}, \ldots, u_{n} \in \mathbb{C}^{*}$ with $\alpha\left(u_{i}, u_{j}\right) \leq \theta$ for all $i, j$ and any $a_{1}, \ldots, a_{n}, b_{1}, \ldots, b_{n} \in S$, the numbers $v=\sum_{i} a_{i} u_{i}$ and $w=\sum_{i} b_{i} u_{i}$ are nonzero and $\alpha(v, w) \leq \phi$. In other words, if $u_{i}$ belong to the angle $|\arg (z)| \leq \theta / 2$ then there exists $\mu \in \mathbb{C}^{*}$ such that $\mu v, \mu w$ belong to the angle $|\arg (u)| \leq \phi / 2$. We say that a set $S \subset \mathbb{C}^{*}$ is $(\theta, \phi)$-angle restricted if $S \in A_{\theta, \phi}$. If $\theta=\phi$ then we denote $A_{\theta, \phi}$ by $A_{\theta}$.

(ii) We denote by $A_{\theta, \phi}^{2}$ the set of subsets $S \subset \mathbb{C}^{*}$ such that for any $a, b, c, d \in S$ the map $z \mapsto \frac{a z+b}{c z+d}$ maps the angle $\left\{z \in \mathbb{C}^{*}:|\arg (z)| \leq \theta\right\}$ into the angle $\left\{u \in \mathbb{C}^{*}:|\arg (u)| \leq \phi\right\}$. In other words, $S \in A_{\theta, \phi}^{2}$ if and only if any $a, b, c, d \in S$ satisfy the condition of (i) for $n=2$. We denote $A_{\theta, \theta}^{2}$ by $A_{\theta}^{2}$.

(iii) We denote by $B_{\theta, \phi}^{2}$ the set of subsets $S \subset \mathbb{C}^{*}$ such that for any $a, b \in S$ the map $z \mapsto \frac{a z+b}{z+1}$ maps the angle $\left\{z \in \mathbb{C}^{*}:|\arg (z)| \leq \theta\right\}$ into the angle $\left\{u \in \mathbb{C}^{*}:|\arg (u)| \leq \phi / 2\right\}$. We denote $B_{\theta, \theta}^{2}$ by $B_{\theta}^{2}$.

Remark. Condition (i) for $n=2$ says that for any $u_{1}, u_{2} \in \mathbb{C}^{*}$ with $\alpha\left(u_{i}, u_{j}\right) \leq \theta$ and $a, b, c, d \in S$ we have $\alpha\left(a u_{1}+b u_{2}, c u_{1}+d u_{2}\right) \leq \phi$. This can be written as $\left|\arg \left(\frac{a z+b}{c z+d}\right)\right| \leq \phi$, where $z:=u_{1} / u_{2}$, which implies that the two definitions of $A_{\theta, \phi}^{2}$ in (ii) are equivalent.

It is clear that $A_{\theta, \phi} \subset A_{\theta, \phi}^{2}$ and $B_{\theta, \phi}^{2} \subset A_{\theta, \phi}^{2}$ (as $\frac{a z+b}{c z+d}=\frac{a z+b}{z+1} \cdot \frac{z+1}{c z+d}$ ), and that $A_{\theta, \phi}, A_{\theta, \phi}^{2}$ are invariant under rescaling by a nonzero complex number, while $B_{\theta, \phi}^{2}$ is invariant under rescaling by a positive real number. Also it is obvious that if $S$ belongs to any of these sets then so do all subsets of $S$. Finally, it is clear that any ray emanating from 0 is in $A_{\theta}$, so we will mostly be interested in sets $S$ that are not contained in a line.

The motivation for studying these notions comes from the following result of A. Barvinok ([B1]).

Theorem 2.2. (i) If $S \in A_{\pi / 2}$ then any square matrix with entries from $S$ has nonzero permanent.

(ii) The disk $|z-1| \leq 1 / 2$ is in $A_{\pi / 2}$. 
This implies that any square matrix with entries $a_{i j}$ such that $\left|a_{i j}-1\right| \leq 1 / 2$ has nonzero permanent. This allowed A. Barvinok to give in [B1] an algorithm for efficient approximate computation of (logarithms of) permanents of such matrices with good precision.

The sets $A_{\theta, \phi}$ for more general $\theta$ and $\phi$, also studied by A. Barvinok, have similar properties and applications (see [B1, B2]). Namely, as explained in [B2], the condition that $S \in A_{\theta, \phi}$ for suitable $\theta$ and $\phi$ guarantees that some quite general combinatorially defined multivariate polynomials $P\left(z_{1}, \ldots, z_{n}\right)$, such as the graph homomorphism partition function, are necessarily non-zero whenever $z_{1}, \ldots, z_{n} \in U$, and can be efficiently approximated there.

The sets $A_{\theta, \phi}^{2}, B_{\theta, \phi}^{2}$ introduced here play an auxiliary role, but they are fairly easy to study (as their definition involves a small number of parameters), and yet we will show that a convex set belonging to $A_{\theta, \phi}^{2}$ must belong to $A_{\theta, \phi}$.

Proposition 2.3. (i) If $S \in A_{\theta, \phi}^{2}$ and $a, b \in S$ then $\alpha(a, b)<\pi-\theta$ and $\alpha(a, b) \leq \phi$.

(ii) If $S \in A_{\theta, \phi}^{2}$ and $a_{1}, \ldots, a_{n} \in S$ then for any $u_{1}, \ldots, u_{n} \in \mathbb{C}^{*}$ with $\alpha\left(u_{i}, u_{j}\right) \leq \theta$ for all $i, j$ we have $\sum_{j} a_{j} u_{j} \neq 0$.

Proof. (i) If $a, b \in S$ then $a u_{1}+b u_{2}$ does not vanish if $\alpha\left(u_{1}, u_{2}\right) \leq \theta$. Suppose $b / a=r e^{i \psi}$ where $0 \leq \psi \leq \pi$ (this can always be achieved by switching $a, b$ if needed). Then $\psi<\pi-\theta$, since otherwise we may take $u_{2}=1, u_{1}=-b / a$ (so that $\alpha\left(u_{1}, u_{2}\right) \leq \theta$ ) and $a u_{1}+b u_{2}=0$, a contradiction. Also $\psi \leq \phi$, since otherwise $\alpha\left(a u_{1}+b u_{2}, a\left(u_{1}+u_{2}\right)\right)$ for $u_{1}=1$ and $u_{2}=N \gg 1$ will exceed $\phi$.

(ii) By (i) we have $\alpha\left(a_{i}, a_{j}\right)<\pi-\theta$ and $\alpha\left(a_{i}, a_{j}\right) \leq \phi<2 \pi / 3$. Thus after rescaling by a complex scalar we may assume that

$$
\left|\arg \left(a_{j}\right)\right|<\frac{1}{2}(\pi-\theta)
$$

for all $j$. Let $u_{1}, \ldots, u_{n} \in \mathbb{C}^{*}$ with pairwise angles $\leq \theta$. By rescaling by a complex scalar we may make sure that $\left|\arg \left(u_{j}\right)\right| \leq \theta / 2$. Then $\left|\arg \left(a_{j} u_{j}\right)\right|<\pi / 2$, so $\operatorname{Re}\left(a_{j} u_{j}\right)>0$ for all $j$. Thus $\sum_{j} a_{j} u_{j} \neq 0$.

Proposition 2.4. Let $\phi \leq \pi / 2$. Then a set $S \subset \mathbb{C}^{*}$ is in $A_{\theta, \phi}^{2}$ if and only if for all $a, b, c, d \in S$ the map $z \mapsto \frac{a z+b}{c z+d}$ maps the angle $\left\{z \in \mathbb{C}^{*}:|\arg (z)| \leq \theta\right\}$ into $\left\{u \in \mathbb{C}^{*}:|\arg (u)| \leq \phi\right\} \cup\{0, \infty\}$.

Proof. Only the "if" direction requires proof. It suffices to show that for $a, b \in S$ and $z \in \mathbb{C}^{*}$ with $|\arg (z)| \leq \theta$ one has $a z+b \neq 0$. Assume the contrary. For any $c \in S$, the map $w \mapsto \frac{a w+b}{c w+c}$ must map the angle $|\arg (z)| \leq \theta$ to the set $\left\{u \in \mathbb{C}^{*}:|\arg (u)| \leq \phi\right\} \cup\{0, \infty\}$, while 
mapping $z$ to 0 . Considering these maps for $c=a, b$ near $w=z$ (with $w / z>0)$ and using that $\phi \leq \pi / 2$, we get that $b / a>0$, i.e., $z<0$, a contradiction.

\section{Convexity And Reduction to $n=2$}

The following theorem reduces checking that a convex set is $(\theta, \phi)$ angle restricted to checking that it is in $A_{\theta, \phi}^{2}$, which is just a lowdimensional geometry problem.

Theorem 3.1. (i) If $S \in A_{\theta, \phi}$ then so is the convex hull of $S$.

(ii) If $S \in A_{\theta, \phi}^{2}$ is convex then $S \in A_{\theta, \phi}$.

Proof. (i) Let $C H(S)$ be the convex hull of $S$. Assume $S \in A_{\theta, \phi}$. Let $a_{1}, \ldots, a_{n}, b_{1}, \ldots, b_{n} \in C H(S)$. Then $a_{i}=\sum_{j} r_{i j} a_{i j}$ where $a_{i j} \in S$, $r_{i j}>0$ and $\sum_{j} r_{i j}=1$. Similarly, $b_{i}=\sum_{k} s_{i k} b_{i k}$ where $b_{i k} \in S, s_{i k}>0$ and $\sum_{k} s_{i k}=1$. Let $u_{1}, \ldots, u_{n} \in \mathbb{C}^{*}$ with angle between each two $\leq \theta$. Let $u_{i j k}=r_{i j} s_{i k} u_{i}$. Consider

$$
v:=\sum_{i, j, k} a_{i j} u_{i j k}=\sum_{i, j, k} a_{i j} r_{i j} s_{i k} u_{i}=\sum_{i, k} a_{i} s_{i k} u_{i}=\sum_{i} a_{i} u_{i}
$$

and

$$
w:=\sum_{i, j, k} b_{i k} u_{i j k}=\sum_{i, j, k} b_{i k} r_{i j} s_{i k} u_{i}=\sum_{i, j} b_{i} r_{i j} u_{i}=\sum_{i} b_{i} u_{i} .
$$

Since $a_{i j}, b_{i k} \in S$, we have that $v, w \neq 0$ and the angle between them does not exceed $\phi$. Thus $C H(S) \in A_{\theta, \phi}$.

(ii) Denote by $R_{n, \theta} \subset \mathbb{C P}^{n-1}$ the set of points $\mathbf{u}=\left(u_{1}, \ldots, u_{n}\right)$ such that the pairwise angles between $u_{i}$ and $u_{j}$ (when both are nonzero) are at most $\theta$. It is clear that $R_{n, \theta}$ is closed (hence compact). By Proposition 2.3(ii) for any $a_{1}, \ldots, a_{n} \in S$ we have $\sum_{j} a_{j} u_{j} \neq 0$. Now fix $a_{1}, \ldots, a_{n}, b_{1}, \ldots, b_{n} \in S$ and consider the function

$$
f\left(u_{1}, \ldots, u_{n}\right)=\operatorname{Im} \log \frac{\sum_{j} a_{j} u_{j}}{\sum_{j} b_{j} u_{j}}
$$

(we choose a single-valued branch of this function). The function $f$ is harmonic on $R_{n, \theta}$ in each variable. Let $\mathbf{u} \in R_{n, \theta}$ be a global maximum or minimum point of $f$. By the maximum principle $2^{2}$, we may choose $\mathbf{u}=\left(u_{1}, \ldots, u_{n}\right)$ so that each $u_{i}$ is zero or has argument $\pm \theta / 2$. By reducing $n$ if needed and relabeling, we may assume that all $u_{j}$ are

\footnotetext{
${ }^{2}$ Note that using the coordinates $v_{i}:=\frac{u_{i}}{\sum_{j=1}^{n} u_{j}}, 1 \leq i \leq n-1$, we may identify $R_{n, \theta}$ with a closed region in $\mathbb{C}^{n-1}$. Thus we may apply the maximum principle for harmonic functions on subsets of a Euclidean space.
} 
nonzero and that $u_{j}=r_{j} e^{i \theta / 2}$ for $j=1, \ldots, m$ and $u_{j}=r_{j} e^{-i \theta / 2}$ for $j=m+1, \ldots, n$, where $r_{j}>0$ for all $j$. By rescaling by a positive real number, we may assume that $\sum_{j=1}^{m} r_{j}=r$ and $\sum_{j=m+1}^{n} r_{j}=1$. Thus we have

$$
v=\sum_{j} a_{j} u_{j}=a r e^{i \theta / 2}+b e^{-i \theta / 2}, \quad w=\sum_{j} b_{j} u_{j}=c r e^{i \theta / 2}+d e^{-i \theta / 2},
$$

where

$$
a=\sum_{j=1}^{m} a_{j} r_{j} / r, b=\sum_{j=m+1}^{n} a_{j} r_{j}, c=\sum_{j=1}^{m} b_{j} r_{j} / r, d=\sum_{j=m+1}^{n} b_{j} r_{j} .
$$

Since $S$ is convex and $a, b, c, d$ are convex linear combinations of the numbers $\left\{a_{j}, j \leq m\right\},\left\{a_{j}, j>m\right\},\left\{b_{j}, j \leq m\right\},\left\{b_{j}, j>m\right\}$ respectively, we get that $a, b, c, d \in S$. Thus, using that $S \in A_{\theta, \phi}^{2}$ and setting $z=r e^{i \theta}$, we see that the angle between $v$ and $w$ does not exceed $\phi$, as claimed.

Lemma 3.2. Let $S \in A_{\theta, \pi / 2}^{2}$, and $a, b \in S$ with $b / a=x+i y, x, y \in \mathbb{R}$. Then we have $x \geq 0$ and

$$
|y| \leq \frac{2 \sqrt{x}+(x+1) \cos \theta}{\sin \theta}
$$

and if $\theta>\pi / 2$ then

$$
\left(x+\frac{1}{\cos \theta}\right)^{2}+y^{2} \leq \tan ^{2} \theta .
$$

In particular, if $\theta>\pi / 2$ then

$$
\frac{1-\sin \theta}{|\cos \theta|} \leq x \leq \frac{1+\sin \theta}{|\cos \theta|}
$$

i.e., $b / a$ is separated from the imaginary axis and from infinity (so any $S \in A_{\theta, \pi / 2}^{2}$ is bounded). Moreover, conditions (1), (2), together with condition (1) with $a$ and $b$ switched are also sufficient for the set $\{a, b\}$ to be in $A_{\theta, \pi / 2}^{2}$.

Proof. Let $a, b \in S$ with $b / a=x+i y$. Pick $u_{1}=r e^{ \pm i \theta}, u_{2}=1$. The angle between $a u_{1}+b u_{2}$ and $a u_{1}+a u_{2}$ does not exceed $\pi / 2$. Hence the real part of $\frac{a u_{1}+b u_{2}}{a u_{1}+a u_{2}}$ is non-negative. Thus, we have

$$
\operatorname{Re}\left(\frac{r e^{ \pm i \theta}+x+i y}{r e^{ \pm i \theta}+1}\right) \geq 0, \forall r>0 .
$$

This yields

$$
\operatorname{Re}\left(\left(r e^{ \pm i \theta}+x+i y\right)\left(r e^{\mp i \theta}+1\right)\right) \geq 0, \forall r>0
$$


i.e.,

$$
r^{2}+((x+1) \cos \theta \pm y \sin \theta) r+x \geq 0, \forall r>0 .
$$

This implies that $x \geq 0$, and minimizing with respect to $r$, we get

$$
(x+1) \cos \theta \pm y \sin \theta \geq-2 \sqrt{x},
$$

which yields

$$
|y| \leq \frac{2 \sqrt{x}+(x+1) \cos \theta}{\sin \theta},
$$

as claimed.

Similarly, the real part of $\frac{a u_{1}+b u_{2}}{b u_{1}+a u_{2}}$ is non-negative. Thus, we have

$$
\operatorname{Re}\left(\frac{r e^{ \pm i \theta}+x+i y}{(x+i y) r e^{ \pm i \theta}+1}\right) \geq 0, \forall r>0 .
$$

This yields

$$
\operatorname{Re}\left(\left(r e^{ \pm i \theta}+x+i y\right)\left((x-i y) r e^{\mp i \theta}+1\right)\right) \geq 0, \forall r>0,
$$

i.e.

$$
x r^{2}+\left(x^{2}+y^{2}+1\right) r \cos \theta+x \geq 0, \forall r>0 .
$$

This is satisfied automatically if $\theta \leq \pi / 2$, but if $\theta>\pi / 2$ then minimizing the left hand side with respect to $r$ gives the condition

$$
\left(x^{2}+y^{2}+1\right) \cos \theta+2 x \geq 0,
$$

which is equivalent to (2).

Finally, to check that $\{a, b\} \in A_{\theta, \pi / 2}^{2}$, it suffices to check that for any $u_{1}, u_{2} \in \mathbb{C}^{*}$ that are within angle $\theta$ of each other, the angles

$\alpha\left(a u_{1}+b u_{2}, a u_{1}+a u_{2}\right), \alpha\left(a u_{1}+b u_{2}, b u_{1}+a u_{2}\right), \alpha\left(a u_{1}+b u_{2}, b u_{1}+b u_{2}\right)$

do not exceed $\pi / 2$. These angles are harmonic functions of $u_{1} / u_{2}$, so the maximum has to be attained on the boundary. Hence it suffices to choose $u_{1}=r e^{ \pm i \theta}$ and $u_{2}=1$. Thus, conditions (1), (2), together with condition (11) with $a$ and $b$ switched are sufficient for the set $\{a, b\}$ to be in $A_{\theta, \pi / 2}^{2}$, as claimed.

Thus we see that the region for $b / a$ is bounded by two parabolas given by (1) and their inversions under the circle $|z|=1$, as well as the circle given by (2) if $\theta>\pi / 2$ (note that this circle is stable under inversion).

Proposition 3.3. Suppose that $\phi \leq \pi / 2$. Then

(i) if $S \in A_{\theta, \phi}^{2}$ then the closure $\bar{S}$ of $S$ in $\mathbb{C}^{*}$ belongs to $A_{\theta, \phi}^{2}$;

(ii) if $S \in A_{\theta, \phi}^{2}$ then the convex hull $C H(S)$ of $S$ belongs to $A_{\theta, \phi}^{2}$. 
Proof. (i) follows by continuity from Proposition 2.4, since the set $\left\{u \in \mathbb{C}^{*}:|\arg (u)| \leq \phi\right\} \cup\{0, \infty\}$ is closed in the Riemann sphere.

(ii) Let $a, b, b^{\prime}, c, d \in \mathbb{C}^{*}$ be such that the maps $z \mapsto \frac{a z+b}{c z+d}$ and $z \mapsto \frac{a z+b^{\prime}}{c z+d}$ satisfy the condition of Proposition 2.4 $r \in[0,1]$ and $b^{\prime \prime}:=r b+(1-r) b^{\prime}$. We claim that the map $z \mapsto \frac{a z+b^{\prime \prime}}{c z+d}$ also satisfies the condition of Proposition 2.4. It suffices to show this for $z \neq-d / c$. We have

$$
\frac{a z+b^{\prime \prime}}{c z+d}=r \frac{a z+b}{c z+d}+(1-r) \frac{a z+b^{\prime}}{c z+d}
$$

and $\frac{a z+b}{c z+d}, \frac{a z+b^{\prime}}{c z+d}$ belong to the set $\left\{u \in \mathbb{C}^{*}:|\arg (u)| \leq \phi\right\} \cup\{0\}$, which is convex since $\phi \leq \pi / 2$. Hence $\frac{a z+b^{\prime \prime}}{c z+d}$ also belongs to this set, as claimed.

Also note that the condition of Proposition 2.4 is invariant under the transpositions $(a, b, c, d) \mapsto(b, a, d, c)$ and $(a, b, c, d) \mapsto(c, d, a, b)$, which generate a group $\mathbb{Z}_{2} \times \mathbb{Z}_{2}$ acting transitively on $a, b, c, d$. Now (ii) follows by using this symmetry and applying the above claim four times (to each of the four variables $a, b, c, d$ ).

This proposition gives a simple method of constructing convex polygons which are in $A_{\theta, \pi}^{2}$ for $\phi \leq \pi / 2$ by doing a finite check on the vertices. We will see examples of this below.

$$
\text { 4. The SETS } A_{\pi / 2}^{2} \text { AND } B_{\pi / 2}^{2}
$$

From now on we focus on the case $\theta=\phi=\pi / 2$ relevant for zero-free regions for the permanent. The general case can be treated by similar methods.

4.1. Explicit characterization. Let us give a more explicit characterization of the sets $A_{\theta}^{2}$ and $B_{\theta}^{2}$ for $\theta=\pi / 2$. Let

$$
F(a, b, c, d)=(\operatorname{Im}(a \bar{d}-b \bar{c}))^{2}-4 \operatorname{Re}(a \bar{c}) \operatorname{Re}(b \bar{d}),
$$

and

$$
G_{1}(a, b)=\left(a_{2}-b_{2}\right)^{2}-4 a_{1} b_{1}, \quad G_{2}(a, b)=\left(a_{1}-b_{1}\right)^{2}-4 a_{2} b_{2},
$$

where $a_{1}+i a_{2}=e^{i \pi / 4} a, b_{1}+i b_{2}=e^{i \pi / 4} b, a_{j}, b_{j} \in \mathbb{R}$. Note that

$$
F(a, b, c, d)=F(b, a, d, c)=F(c, d, a, b)=F(d, c, b, a) .
$$

Lemma 4.1. (i) $S \in A_{\pi / 2}^{2}$ if and only if for any $a, b, c, d \in S$ we have $F(a, b, c, d) \leq 0$.

(ii) $S \in B_{\pi / 2}^{2}$ if and only if $|\arg (a)| \leq \pi / 4$ for $a \in S$, and for any $a, b \in S$ we have $G_{1}(a, b) \leq 0, G_{2}(a, b) \leq 0$. 
Proof. (i) Suppose that $F(a, b, c, d) \leq 0$ for all $a, b, c, d \in S$. Then $\operatorname{Re}(a \bar{c}) \geq 0$ for all $a, c \in S$ (as we can take $b=d$ ). Therefore, $\frac{a z+b}{c z+d} \neq 0$ when $\operatorname{Re}(z) \geq 0$. Indeed, otherwise, we must have $\operatorname{Re}(b / a)=$ $|a|^{-2} \operatorname{Re}(b \bar{a}) \leq 0$, so $\operatorname{Re}(b / a)=0$ and $b / a=i t$ for some real $t \neq 0$. But then $F(a, b, a, a)=t^{2}|a|^{4}>0$, a contradiction.

Thus by the definition of $A_{\pi / 2}^{2}$, it suffices to show that for $a, b, c, d \in S$ one has $\operatorname{Re} \frac{a z+b}{c z+d} \geq 0$ whenever $z=i t, t \in \mathbb{R}$. We have

$$
\frac{a i t+b}{c i t+d}=\frac{(a i t+b)(-\bar{c} i t+\bar{d})}{|c i t+d|^{2}}
$$

and

$$
\operatorname{Re}((a i t+b)(-\bar{c} i t+\bar{d}))=\operatorname{Re}(a \bar{c}) t^{2}-\operatorname{Im}(a \bar{d}-b \bar{c}) t+\operatorname{Re}(b \bar{d}) .
$$

Since $\operatorname{Re}(a \bar{c}), \operatorname{Re}(b \bar{d}) \geq 0$, the condition for this to be $\geq 0$ is that the discriminant of this quadratic function is $\leq 0$, which gives the result.

Conversely, if $S \in A_{\pi / 2}^{2}$ then the above calculation shows that $F(a, b, c, d) \leq 0$ for all $a, b, c, d \in S$.

(ii) Let $a^{\prime}=e^{i \pi / 4} a=a_{1}+i a_{2}, b^{\prime}=e^{i \pi / 4} b=b_{1}+i b_{2}$. The condition on $a^{\prime}, b^{\prime}$ is that for $t \in \mathbb{R}$ we have $\operatorname{Re} \frac{a^{\prime} i t+b^{\prime}}{i t+1} \geq 0$ and $\operatorname{Im} \frac{a^{\prime} i t+b^{\prime}}{i t+1} \geq 0$. We have

and

$$
\frac{a^{\prime} i t+b^{\prime}}{i t+1}=\frac{\left(a^{\prime} i t+b^{\prime}\right)(-i t+1)}{t^{2}+1},
$$

$$
\begin{gathered}
\left(a^{\prime} i t+b^{\prime}\right)(-i t+1)=a^{\prime} t^{2}+\left(a^{\prime}-b^{\prime}\right) i t+b^{\prime}= \\
=\left(a_{1} t^{2}-\left(a_{2}-b_{2}\right) t+b_{1}\right)+i\left(a_{2} t^{2}+\left(a_{1}-b_{1}\right) t+b_{2}\right) .
\end{gathered}
$$

Since $a_{1}, a_{2}, b_{1}, b_{2} \geq 0$ (as seen by setting $t=0$ and $t=\infty$ ), the condition is that the discriminants of these two quadratic functions must be $\leq 0$, which gives the result.

\subsection{Examples.}

Example 4.2. Lemma 4.1(ii) implies that the interval $[a, b] \subset \mathbb{R}$ for $0<a \leq b$ is in $B_{\pi / 2}^{2}$ iff $b / a \leq 3+2 \sqrt{2}$.

Example 4.3. Let $a=1 / 2, b=1+i / 2, c=1-i / 2$ and $d=3 / 2+t$. Let us find the largest $t>0$ for which $\{a, b, c, d\}$ is in $B_{\pi / 2}^{2}$ (hence in $\left.A_{\pi / 2}^{2}\right)$. Since $a, b, c$ belong to the disk $|z-1| \leq 1 / 2$, which was shown by A. Barvinok in [B1] to belong to $B_{\pi / 2}^{2}$, it suffices to check when $G_{i}(a, d) \leq 0, G_{i}(b, d) \leq 0, G_{i}(c, d) \leq 0$. The first condition gives the inequality of Example 4.2 , which is $3+2 t \leq 3+2 \sqrt{2}$, i.e. $t \leq \sqrt{2}$. The second (or, equivalently, third) condition gives the inequalities $t^{2} \leq 2 t+3,(1+t)^{2} \leq 3(2 t+3)$ which hold for $0 \leq t \leq \sqrt{2}$. Thus 
we find that the optimal value is $t=\sqrt{2}$ and the quadrilateral with vertices $1 / 2,1 \pm i / 2$ and $\frac{3}{2}+\sqrt{2}$ :

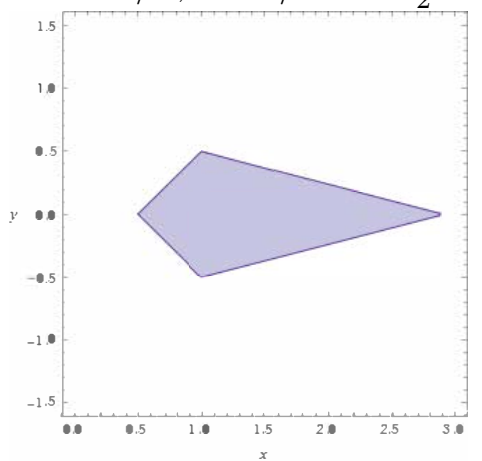

is in $A_{\pi / 2}^{2}$, hence in $A_{\pi / 2}$ by Proposition 3.3 (ii); thus it is a zero-free region for the permanent.

Example 4.4. Let us find the values of $t>1 / 2$ for which the union of the disk $|z-1| \leq 1 / 2$ and the point $1+t$ belongs to $B_{\pi / 2}^{2}$ (hence to $A_{\pi / 2}^{2}$ ). Such $t$ are determined by the condition that $G_{1}\left(1+\frac{1}{2} e^{i(\phi-\pi / 4)}, 1+t\right) \leq 0$ for all $\phi$ (the condition involving $G_{2}$ is the same due to axial symmetry). This can be written as

$$
\left(t+\frac{1}{\sqrt{2}} \cos \phi\right)^{2} \leq 4(1+t)\left(1+\frac{1}{\sqrt{2}} \sin \phi\right)
$$

for all $\phi$. This gives

$t \leq 2+\sqrt{2} \sin \phi-\frac{\sqrt{2}}{2} \cos \phi+\sqrt{6 \sqrt{2} \sin \phi-2 \sqrt{2} \cos \phi-\sin 2 \phi-\cos 2 \phi+9}$, and minimizing this function (numerically), we get the answer

$$
t \leq t_{*}=1.64 \ldots
$$

Thus the ice cream cone, which is the convex hull of the disk $|z-1| \leq$ $1 / 2$ and the point $1+t_{*}$ (significantly larger than the disk):

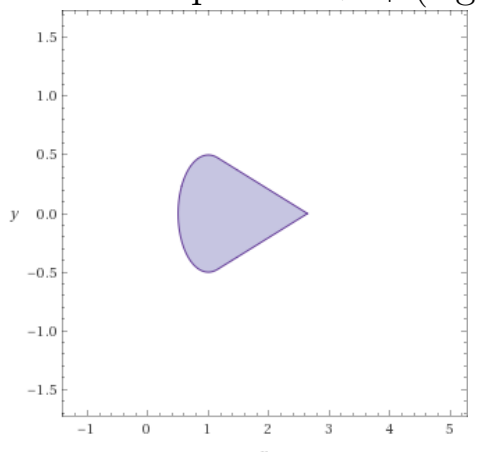

belongs to $A_{\pi / 2}^{2}$, hence to $A_{\pi / 2}$ by Proposition 3.3 (ii), and thus is a zero-free region for the permanent. 
Example 4.5. Let $S=\{a, b\}$, and $b / a=x+i y$. Let us compute when $S \in A_{\pi / 2}^{2}$. By Lemma 3.2 the conditions for this are

$$
y^{2} \leq 4 x, \quad y^{2} \leq 4 x\left(x^{2}+y^{2}\right)
$$

This gives

$$
|y| \leq 2 \sqrt{x} ; \text { and }|y| \leq \frac{2 x^{3 / 2}}{\sqrt{1-4 x}}, x<1 / 4
$$

So we get a region which is bounded by a parabola and its inversion with respect to the circle $|z|=1$, which is a cissoid of Diocles:

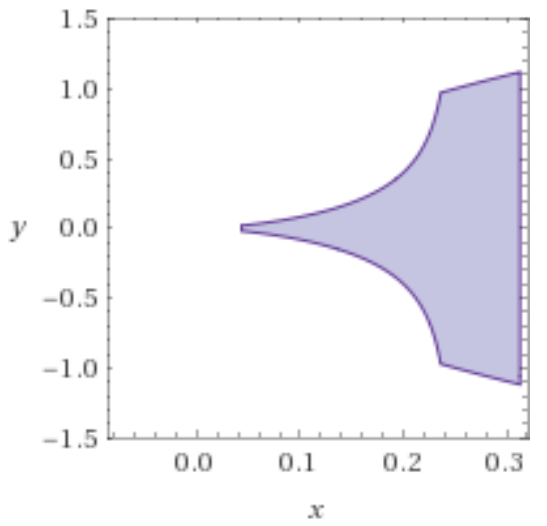

By Proposition 3.3, this is also the necessary and sufficient condition for the segment $[a, b] \subset \mathbb{C}^{*}$ to be in $A_{\pi / 2}^{2}$.

Example 4.6. Consider now a 3-element set $S=\{1, a, b\}$ and let us give a necessary condition for it to be in $A_{\pi / 2}^{2}$.

Proposition 4.7. Assume $a \notin \mathbb{R}$. Then one has

$$
a_{1} \frac{\left(|1+a|-1-a_{1}\right)^{2}}{a_{2}^{2}} \leq b_{1} \leq a_{1} \frac{\left(|1+a|+1+a_{1}\right)^{2}}{a_{2}^{2}},
$$

where $a=a_{1}+i a_{2}, b=b_{1}+i b_{2}$ and $a_{1}, a_{2}, b_{1}, b_{2} \in \mathbb{R}$. In other words, one has $K^{-1} \leq \frac{b_{1}}{a_{1}} \leq K$, where $K:=\frac{\left(|1+a|+1+a_{1}\right)^{2}}{a_{2}^{2}}$. Thus any $S \in A_{\pi / 2}^{2}$ which is not contained in a line is bounded and separated from the origin.

Proof. We have the inequalities $F(a, 1,1, b) \leq 0$ and $F(a, b, 1,1) \leq 0$, which yields

$$
\left(a_{1} b_{2}-a_{2} b_{1}\right)^{2} \leq 4 a_{1} b_{1}, \quad\left(a_{2}-b_{2}\right)^{2} \leq 4 a_{1} b_{1},
$$

or, equivalently,

$$
\left|a_{1} b_{2}-a_{2} b_{1}\right| \leq 2 \sqrt{a_{1} b_{1}}, \quad\left|a_{2}-b_{2}\right| \leq 2 \sqrt{a_{1} b_{1}} .
$$


From the second inequality in 4 we have

$$
\left|b_{2}\right| \leq 2 \sqrt{a_{1} b_{1}}+\left|a_{2}\right| .
$$

Hence

$$
\left|a_{1} b_{2}\right| \leq\left(2 \sqrt{a_{1} b_{1}}+\left|a_{2}\right|\right) a_{1} .
$$

Thus

$$
\left|a_{2} b_{1}\right| \leq 2 \sqrt{a_{1} b_{1}}+\left|a_{1} b_{2}\right| \leq 2\left(1+a_{1}\right) \sqrt{a_{1} b_{1}}+\left|a_{2}\right| a_{1} .
$$

Hence

$$
b_{1} \leq \frac{2\left(1+a_{1}\right) \sqrt{a_{1} b_{1}}}{\left|a_{2}\right|}+a_{1}
$$

This yields

$$
b_{1} \leq a_{1} \frac{\left(|1+a|+1+a_{1}\right)^{2}}{a_{2}^{2}},
$$

as claimed. From this we also have

$$
\left|a_{2}\right| \leq 2 \sqrt{a_{1} b_{1}}+\left|b_{2}\right| \leq 2 \sqrt{a_{1} b_{1}}+\frac{2 \sqrt{a_{1} b_{1}}+\left|a_{2}\right| b_{1}}{a_{1}},
$$

which yields

$$
b_{1} \geq \frac{a_{1}\left(|1+a|-1-a_{1}\right)^{2}}{a_{2}^{2}},
$$

again as claimed. Now (5) implies that $S$ is bounded if it is not contained in a line.

4.3. Rectangular and trapezoidal regions. Let us now try to characterize rectangular and trapezoidal regions which are in $A_{\pi / 2}^{2}$ (hence in $\left.A_{\pi / 2}\right)$.

Proposition 4.8. (i) Let $L, M, N>0$ and $R(M, L, N)$ be the rectangle $M \leq x \leq M+L,|y| \leq N$. Then $R(M, L, N) \in A_{\pi / 2}^{2}$ if

$$
N \leq \frac{2 M^{3 / 2}}{\sqrt{L+24 M}} .
$$

(ii) Let $0<M<L$ and $T(M, L, t)$ be the trapezoid

$$
M \leq x \leq L,|y| \leq t x .
$$

Then $T(M, L, t) \in A_{\pi / 2}^{2}$ if $t<\sqrt{2}-1$ and

$$
\begin{gathered}
L \leq M\left(\frac{t^{2}+t^{-2}-4+\left(t^{-1}-t\right) \sqrt{t^{2}+t^{-2}-6}}{2}\right)^{1 / 2} \\
=M t^{-1}(1+o(t)) \text { as } t \rightarrow 0 .
\end{gathered}
$$


Proof. In coordinates the desired basic inequality $F(a, b, c, d) \leq 0$ looks like

$$
\left(a_{2} d_{1}-a_{1} d_{2}-b_{2} c_{1}+b_{1} c_{2}\right)^{2} \leq 4\left(a_{1} c_{1}+a_{2} c_{2}\right)\left(b_{1} d_{1}+b_{2} d_{2}\right),
$$

where the subscript 1 denotes the real part and the subscript 2 the imaginary part (i.e., $a_{1}=\operatorname{Re}(a), a_{2}=\operatorname{Im}(a)$ etc.).

(i) Since the absolute values of $a_{2}, b_{2}, c_{2}, d_{2}$ don't exceed $N$, the basic inequality would follow from the inequality

$$
\begin{gathered}
N^{2}\left(a_{1}+b_{1}+c_{1}+d_{1}\right)^{2} \leq 4\left(a_{1} c_{1}-N^{2}\right)\left(b_{1} d_{1}-N^{2}\right)= \\
=4 a_{1} c_{1} b_{1} d_{1}-4 N^{2}\left(a_{1} c_{1}+b_{1} d_{1}\right)+N^{4} .
\end{gathered}
$$

(as long as $N \leq M$, which follows from the inequality in (i)). This, in turn, would follow from the inequality

$$
N^{2}\left(\left(a_{1}+b_{1}+c_{1}+d_{1}\right)^{2}+4\left(a_{1} c_{1}+b_{1} d_{1}\right)\right) \leq 4 a_{1} c_{1} b_{1} d_{1} .
$$

Let $q$ be the largest of $a_{1}, b_{1}, c_{1}, d_{1}$ and $p$ the second largest. Then the latter inequality would follow from the inequality

$$
N^{2}\left(\left(a_{1}+b_{1}+c_{1}+d_{1}\right)^{2}+4\left(a_{1} c_{1}+b_{1} d_{1}\right)\right) \leq 4 M^{2} p q .
$$

Now observe that on the left hand side we have 24 quadratic monomials in $a_{1}, b_{1}, c_{1}, d_{1}$, which are all $\leq p q$ except one, which is $q^{2} \leq(M+L) q$. So the last inequality would follow from the inequality

$$
N^{2}(23 p+M+L) \leq 4 M^{2} p,
$$

or

$$
N^{2}(M+L) \leq p\left(4 M^{2}-23 N^{2}\right) .
$$

This, in turn, follows from the inequality

$$
N^{2}(M+L) \leq M\left(4 M^{2}-23 N^{2}\right),
$$

or

$$
N^{2}(L+24 M) \leq 4 M^{3},
$$

giving

$$
N \leq \frac{2 M^{3 / 2}}{\sqrt{L+24 M}}
$$

as claimed.

(ii) Since $\left|a_{2}\right| \leq t a_{1},\left|b_{2}\right| \leq t b_{1},\left|c_{2}\right| \leq t c_{1},\left|d_{2}\right| \leq t d_{1}$, the basic inequality would follow from the inequality

$$
4 t^{2}\left(a_{1} d_{1}+b_{1} c_{1}\right)^{2} \leq 4\left(1-t^{2}\right)^{2} a_{1} c_{1} b_{1} d_{1},
$$

which is equivalent to the inequality

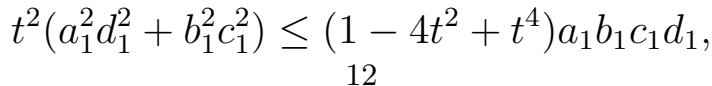


or $\mu+\frac{1}{\mu} \leq t^{-2}-4+t^{2}$, where $\mu=\frac{a_{1} d_{1}}{b_{1} c_{1}}$. The largest value of this ratio is $L^{2} / M^{2}$, so it sufficient to require that

$$
\frac{L^{2}}{M^{2}}+\frac{M^{2}}{L^{2}} \leq t^{2}-4+t^{-2}:=T \text {. }
$$

This is satisfied whenever

$$
L \leq M\left(\frac{T+\sqrt{T^{2}-4}}{2}\right)^{1 / 2}
$$

as claimed.

In particular, if $L=1$ and $M$ is small then for the rectangle we have $N=2 M^{3 / 2}(1+o(M))$. Comparing this to the bound (3), we see that this is sharp up to a factor $1+o(M)$. This also relaxes the bound $N \leq C M^{2}$ from [B1].

Also for the trapezoid we have $M \geq t(1+o(t))$, so its short side has half-length $N=t M$, so the largest possible $N$ is $\sim M^{2}$.

4.4. Maximal angle-restricted sets. From now on we will only consider closed convex sets $S$, since we have seen in Proposition 3.3 that if $S \in A_{\pi / 2}^{2}$ then so do its closure and its convex hull, and a convex set is in $A_{\pi / 2}$ iff it is in $A_{\pi / 2}^{2}$.

It is clear from Zorn's lemma that any $(\pi / 2, \pi / 2)$-angle restricted set is contained in a maximal one, which is necessarily closed and convex. The problem of finding and classifying maximal $(\pi / 2, \pi / 2)$-anglerestricted sets is a special case of a more general problem of optimal control theory - to find maximal regions $R$ with the property that a given function $F\left(z_{1}, \ldots, z_{n}\right)$ is $\leq 0$ when all $z_{i} \in R$; one of the simplest and best known problems from this family is to describe curves of constant width $\ell$ (in this case $\left.F\left(z_{1}, z_{2}\right)=\left|z_{1}-z_{2}\right|^{2}-\ell^{2}\right)$. As is typical for such problems, the problem of describing maximal regions in $A_{\pi / 2}$ is rather nontrivial; presumably, it can be treated by the methods of the book BCGGG.

Maximal regions can also be constructed as limits of nested sequences $\Pi_{n}$ of convex $n$-gons, each obtained from the previous one by "pushing out" a point on one of the sides as far as it can go while still preserving the property of being in $A_{\pi / 2}$. This approach should be good for numerical computation of maximal regions, since the verification that the region is in $A_{\pi / 2}^{2}$ (equivalently, in $A_{\pi / 2}$ ) is just a finite check on the vertices of the polygon.

Here we will not delve into this theory and will restrict ourselves to proving the following result. Let $\mu_{S}(a):=\max _{b, c, d \in S} F(a, b, c, d)$. We have seen that $S \in A_{\pi / 2}$ iff $\mu_{S}(a) \leq 0 \forall a \in S$. 
Proposition 4.9. A closed convex set $S \in A_{\pi / 2}$ (not contained in a line) is maximal iff $\mu_{S}(a)=0$ for all $a \in \partial S$.

Proof. Note that $S$ is bounded by Proposition 4.7, hence compact. Suppose $S \in A_{\pi / 2}$ is maximal and $a \in \partial S$ is such that there are no $b, c, d \in S$ with $F(a, b, c, d)=0$. Then $\mu_{S}(a)=-\varepsilon<0$. Now take sufficiently small $\delta$ and let $S^{\prime}=S \cup\{|z-a| \leq \delta\}$, which is strictly larger than $S$ as $a \in \partial S$. Let us maximize $F(x, b, c, d)$ over $x, b, c, d \in$ $S^{\prime}$. If these points are further than $\delta$ from $a$ then they are in $S$ so $F(x, b, c, d) \leq 0$. Otherwise, if one of them is $\delta$-close to $a$, say, $x$ (it does not matter which one because of the permutation symmetry of $F$ ), then $F(x, b, c, d) \leq F(a, b, c, d)+\varepsilon \leq 0$ (a number $\delta$ with this property exists due to uniform continuity of $F$ on $S)$. So $S^{\prime}$ and its convex hull are in $A_{\pi / 2}$, contradicting the assumption that $S$ is maximal.

Conversely, suppose $\mu_{S}(a)=0$ on $\partial S$, let $S^{\prime} \supset S$ be a larger convex region. Then there exists $a \in \partial S$ which is an interior point of $S^{\prime}$. Also there exist $b, c, d \in S$ with $F(a, b, c, d)=0$. But for fixed $b, c, d$ the function $F(z, b, c, d)$ is inhomogeneous quadratic in $z, \bar{z}$ with nonnegative degree 2 part, which implies that there is a point $a^{\prime}$ arbitrarily close to $a$ with $F\left(a^{\prime}, b, c, d\right)>0$. Hence $S^{\prime} \notin A_{\pi / 2}$ and $S$ is maximal.

Thus, we see that if $S \in A_{\pi / 2}$ and $a \in \partial S$ with $\mu_{S}(a)<0$ then $S$ can be enlarged near $a$ (e.g. by adding a point $a^{\prime} \notin S$ close to $a$ and taking the convex hull of $S$ and $a^{\prime}$ ), so that the larger set $S^{\prime}$ is still in $A_{\pi / 2}$. Otherwise, if $\mu_{S}(a)=0$, then $a$ must be on the boundary of any $S^{\prime} \in A_{\pi / 2}$ containing $S$. We will say that $S$ is maximal at $a$ if $\mu_{S}(a)=0$ and non-maximal at $a$ if $\mu_{S}(a)<0$.

Example 4.10. Let $S$ be the disk $|z-1| \leq 1 / 2$. Then it is easy to check that $S$ is maximal at the three points $a=1 / 2,1 \pm i / 2$ (indeed, picking $b, c, d$ from the same set, we can make $F(a, b, c, d)=0)$. On the other hand, we claim that $S$ is not maximal at any other points of the boundary circle. The proof is by a direct computation. Namely, if $a \neq 1 / 2,1 \pm i / 2$ but $|a-1|=1 / 2$, then it can be shown that for any $b$ with $|b-1| \leq 1 / 2$ one has $G_{1}(a, b)<0$ and $G_{2}(a, b)<0$ (hence, any small perturbation of $S$ at $a$ will still be in $B_{\pi / 2}^{2}$, hence in $\left.A_{\pi / 2}^{2}\right)$. Indeed, setting $a=1+\frac{1}{2} e^{i(u-\pi / 4)}$ and $b=1+\frac{1}{2} e^{i(v-\pi / 4)}$ for $u, v \in \mathbb{R} / 2 \pi \mathbb{Z}$, we have

$$
G_{1}(a, b)=H(u, v):=\frac{1}{4}(\sin u-\sin v)^{2}-(\sqrt{2}+\cos u)(\sqrt{2}+\cos v),
$$

and maximization of this function (e.g., using Wolfram Alpha, or analytically) yields $H(u, v) \leq 0$, and $H(u, v)=0$ if and only if $u=-3 \pi / 4$ 
and $v=3 \pi / 4$ or $u=3 \pi / 4$ and $v=-3 \pi / 4$, which implies the desired statement.

\section{REFERENCES}

[B1] A. Barvinok, Approximating permanents and Hafnians, Discrete analysis, 2017:2, 34 pp.

[B2] A. Barvinok, Combinatorics and complexity of partition functions, Springer, 2016.

[BCGGG] R.L. Bryant, S.S. Chern, R.B. Gardner, H.L. Goldschmidt, and P.A. Griffiths, Exterior Differential Systems. Springer, 2011.

[JSV] M. Jerrum, A. Sinclair, E. Vigoda, A Polynomial-Time Approximation Algorithm for the Permanent of a Matrix with Nonnegative Entries, Journal of the ACM, Volume 51, Issue 4, July 2004, Pages 671-697. 